\title{
Reconocimiento: perfiles ético-políticos de una categoría en proceso de redefinición ${ }^{1}$
}

\author{
MARÍA JOSÉ GUERRA PALMERO \\ Universidad de La Laguna
}

El tópico del RECONOCIMIENTO HA EMERGido cON FUERZa en los noventa. Su genealogía histórico-filosófica se remonta a una de esas sendas perdidas en las que se adentró el joven Hegel en la época de Jena para después abandonarla. Habermas topa con el motivo del reconocimiento y lo utiliza para impulsar el giro intersubjetivo y su trama pragmática: es una pieza básica en la dinámica de la individualización que es, también, socialización. La formulación de Mead del kotro generalizado» le permitirá convertirlo en clave de su abstracta y formalizada propuesta ético discursiva. No obstante, al dar cuenta de las discusiones en torno al motivo del reconocimiento y a su relevancia ética-política no podemos obviar el humus del que parte la reactivación actual de la categoría: los debates sobre el multiculturalismo y las demandas políticas de los feminismos en el contexto de las denominadas políticas de la identidad y de las diferencias.

Taylor, desde su comunitarismo neohegeliano, enfrentando la espinosa cuestión de los derechos colectivos, acuña, la formulación «política

1 Este trabajo se ha realizado con la financiación del proyecto de la DGICYT PB97-1473. 
del reconocimiento», pero ya antes, en el año 90, Iris M. Young desafiaba la comprensión estrecha de la justicia como mera redistribución al pretender desentrañar la implícita teoría de la justicia que está detrás de la práctica de movimientos sociales tales como el feminismo, el antirracismo, los protagonizados por gays y lesbianas y el multiculturalismo y su demanda de reconocimiento de las diferencias. En los últimos tiempos, Nancy Fraser ha propuesto, en un ensayo que ha impactado con fuerza la conciencia de las izquierdas norteamericanas, una teoría bifronte de la justicia que de cuenta de las complejidades e intersecciones entre las desigualdades sociales y los déficits de reconocimiento que sufren determinados grupos. Nuestro modesto objetivo en estas páginas es preguntarnos por la pertinencia y cabida de la noción de reconocimiento en el terreno político. Taylor y Young, por poner dos ejemplos que tiran en direcciones distintas, promueven un cambio de paradigma en teoría política al objetar los excesos individualistas liberales. A esta luz nos podemos hacer las siguientes preguntas: ¿Qué efectos debe tener en nuestra comprensión de la política el giro intersubjetivo? ¿Cuáles son las entidades con las que debemos tratar: individuos o grupos o de los dos en sus mediaciones? ¿Cómo tratar con las aspiraciones políticas de las identidades (hacia dentro) - diferencias (hacia fuera)? ¿Cómo trasladar a la política una noción de reconocimiento cuya génesis es marcadamente moral?

El protagonismo moral de la categoría de reconocimiento queda probado fácilmente. Es el fundamento sobre el que se construye la identidad moral de los individuos. Tanto la autonomía como la autorrealización -intensificada por el giro expresivista como tarea moral-requieren de autoconfianza, autorrespeto y autoestima. La red intersubjetiva que prodiga reconocimiento, a través de las más variadas formas del vínculo -amor, respeto y solidaridad-, es condición de posibilidad de la misma subjetividad moral. Y, a esta luz, la moralidad misma aparece como una estructura cuyo objetivo es salvaguardar la integridad de las personas al tomar nota del dato esencial de su vulnerabilidad, de su fragilidad. Pero, ¿quiere esto decir que debemos proteger todo mundo de vida, toda comunidad debido a su condición fundante de la posibilidad de despegue de toda identidad individual?

La protección de las comunidades tradicionales nos plantea el problema de posibles incompatibilidades entre la defensa de las diferencias y la salvaguarda de la justicia y de los mismos derechos individuales. Las tensiones entre multiculturalismo y feminismo hablan de este hecho. Pero, además, la correlación comunidad-identidad-diferencias nos enfrenta directamente con el antagonismo de la construcción nosotros/ 
ellos en la que anidan las consecuencias excluyentes y marginalizadoras que en muchas ocasiones promueven las comunidades ya constituidas frente a los percibidos como «ajenos». Tanto hacia dentro como hacia fuera no podemos suponer la inocencia del grupo o la comunidad tan sólo por su papel indudable de sostén de las individualidades. Sin el reconocimiento que se forja en las comunidades la emergencia de las identidades sería impensable, pero los modos de este reconocimiento y la estructuración de esas comunidades debe estar sometida a crítica. Esta posibilidad sólo viene dada al atender el punto de vista de los outsiders o de aquellos que transitan entre distintas comunidades y cuyas identidades-puente se convierten así no sólo en más complejas sino, también, en mediadoras.

Desde el punto de vista de Honneth, el protagonismo político del reconocimiento deriva de su poder motivador para impulsar las exigencias y demandas que objetan el menosprecio, la humillación y la crueldad destinada a determinados grupos sociales devaluados y desestimados por la sociedad. El reconocimiento es así la clave de la gramática moral de los conflictos sociales. La aproximación al carácter moral del reconocimiento que impulsa a Honneth es negativa: la «fenomenología del desprecio». Se trata de detectar el fenómeno a partir de las dis-funciones que crean la humillación y la violencia como desgarrones en la red moral que nos sostiene. Margalit sitúa el asunto del reconocimiento en un territorio social, institucional, pero más que suponer que el reconocimiento es una de las caras de la justicia, suma a ésta otra nota deseable para la convivencia social: la decencia. Esta consistiría en que las instituciones no humillaran a los individuos.

En lo que viene a continuación y dando por supuesto el acierto y la centralidad moral del reconocimiento voy a intentar presentar algunos de los problemas de su traslación al ámbito político como reconocimiento de las diferencias. A propósito de este asunto voy a hacer una breve cala en la polémica Young-Fraser.

\section{I. ¿DE QUÉ DIFERENCIAS ESTAMOS HABLANDO?}

En las afirmaciones de la política de las diferencias no podemos dejar que todos los gatos sean pardos. La alternativa que va a ofrecer Fraser exige una «diferenciada política de las diferencias», esto es, no todas pueden ser tratadas por igual y con el mismo respeto. Un pluralismo irrestricto sin límites no es posible pensarlo. Por ejemplo el modelo que Young privilegia en su análisis del año 90 es el del grupo cultural. Esto lleva a minimizar la atención a la conexión entre desigualdad y diferen- 
cias porque a veces «la justicia puede requerir precisamente reducir la diferenciación del grupo al, por ejemplo, reestructurar la división del trabajo» (Fraser 1997, p. 196). Pero, también, ofrece otra clara desventaja porque no se ve, no queda claro como es posible incorporar a esta comprensión de las identidades una dimensión autocrítica. El mero respeto a las diferencias apuntala la fijeza de las identidades dadas y parece no dejar espacio para la autotransformación o la redefinición de los patrones de autoidentificación de individuos y grupos.

La consideración de las diferencias necesita de más rigor analítico. Los vectores de discriminación social relacionados con el género, la raza, la cultura o la orientación sexual requieren, a su vez, de análisis diferenciados para posibilitar, después, la comprensión de sus cruces e interacciones. Las tensiones entre las demandas de la redistribución y el reconocimiento nos enfrentan a interrogantes acerca de qué diferencias debemos preservar y cuáles sacrificar si se demuestra que dependen de modos de opresión tales como la explotación o la marginalización. Por otra parte, Fraser sugiere que habrá diferencias ante las cuales el mero pensar en su afirmación nos resultará repulsivo si representa puntos de vista excluyentes y violentos -pone el ejemplo de los neo-nazis skinhead-, pero, igualmente, podríamos pensar en tipos de nacionalismo, más aclimatados en la vieja Europa que defienden definiciones biologicistas y puristas de su identidad étnico-nacional.

En suma, frente a la propuesta de Young, Fraser acaba proponiendo que las diferencias no pueden ser sólo entendidas como «simplemente variaciones» que «no deberían ser ni eliminadas ni universalizadas sino más bien afirmadas como diferencias; valoradas como expresiones de la diversidad humana» (Fraser 1997, p. 204). Su opción es más compleja y matizada porque, en primer lugar, las diferencias causadas por la desigualdad económica deberán desaparecer en cuanto ésta sea inhabilitada, en segundo lugar, las que no dependan de factores económicos y hayan sido marginadas por los standares de los grupos dominantes podrán aspirar a la universalización, y en tercer lugar, todavía nos quedarán otras diferencias sobre las que afirmaremos con Young que deben ser, meramente, respetadas en su diversidad. Esta triple opción clasificatoria indica que Fraser se atreve a introducir un criterio normativo a la hora de discernir entre el carácter de las diferencias. Discriminar entre las pretensiones de validez de las diferencias será una tarea más para la plural, inclusiva y abierta esfera dialógica con la que ella identifica el deber ser de la política. La teoría crítica del reconocimiento que Fraser va a impulsar estará atenta a distinguir qué demandas del reconocimiento de la diferencia contribuyen al avance de la igualdad social y cuáles la retrasan o la socavan (Fraser 1997, p. 5). 
Young, en su último libro Intersecting Voices. Moral Dilemmas of Gender, Political Philosophy and Policy, sin renunciar a su convicción del respeto a las diferencias, las somete a un proceso de «pragmatización» en la que la posibilidad crítica queda abierta a través de su explicitación en el diálogo. Young sigue el camino de apostillar, remozar y mejorar el modelo de democracia deliberativa -inspirado por Habermas y Arendt y corregido, en este caso, por Benhabib- que es el auténtico caballo de batalla de las derivas teórico-políticas a las que estamos aludiendo. Como apuntaré al final el tratamiento pragmático de la cuestión de las identidades-diferencias y de sus demandas políticas merece ser tenido en cuenta a la hora de introducir mecanismos y procedimientos que corroboren el reconocimiento de las personas y de sus diferencias.

\section{RECONOCIMIENTO Y CIUDADANÍA PLENA}

Parte de lo que se denuncia en las intervenciones a favor de la traslación política de la categoría reconocimiento es que la cultura dominante es un «locus de opresión» que exige que los individuos se asimilen a un canon normalizado y que silencien su identidad especifica y diferenciada. La imparcialidad de la cultura dominante es tan sólo un mito que tritura las diferencias. En mi modesta interpretación el tópico del reconocimiento, en sus connotaciones más políticas, se enfrenta a los efectos de una perversa dialéctica de la ilustración -relativa al uso y abuso de las abstracciones-que logró identificar de manera subrepticia y velada igualdad con asimilación, homogeneidad o uniformización canonizando un modelo de ciudadano -muy semejante al burgués- del que quedaban excluidos los y las diferentes. Hans Mayer en su Historia de la literatura maldita aporta sustancia literaria y política a este efecto que recorre, especialmente, el siglo XIX: las mujeres, los judíos o los homosexuales son aquellos a quienes se consideraba «marginados existenciales». El problema no está en lo que hacen sino en lo que son, su culpa es ontológica. Tales marginados quedaban, en consecuencia, privados tanto de la estima social como de la dignidad de la ciudadanía o, como mal menor, condenados a contentarse con una ciudadanía precaria de segunda o tercera clase.

A esta luz, el reconocimiento de los individuos se revela en sus diversas dimensiones como condición de posibilidad de ingreso con garantías en el ámbito discursivo y representativo de la política. El reconocimiento va a ser sumado a la demanda de igualdad de oportunidades que exige la definición social de la ciudadanía como condición de posibilidad de una democracia radical. De una lado, las exigencias de la 
izquierda cultural y del multiculturalismo, que aquí hemos señalado al referirnos a la obra de Young, y del otro los desarrollos teóricos de rescate y reformulación de la idea de reconocimiento, van a replantear las bases mismas de la ciudadanía atendiendo a la «injusticia cultural».

La injusticia cultural tiene efectos normativos y axiológicos. De un lado, se excluye a los que son percibidos como «diferentes», del otro se los desvaloriza, con lo que el maltrato normativo y axiológico se refuerzan. De hecho las injusticias culturales suelen anudarse con las desigualdades socioeconómicos y con los déficits de representación política. El concepto acuñado por Bourdieu de «violencia simbólica» es pertinente aquí para abordar con garantías los efectos y formas de proceder de la injusticia cultural que no es sino otro modo de la opresión. El caso es que hace falta ir más allá de un paradigma redistributivo estrecho que no contemple los mecanismos por los que se suelen reforzar los vectores de discriminación. No podemos, además, suponer que todas las diferencias son producidas por las desigualdades económicas y sociales. Necesitamos una intelección «diferenciada» de la naturaleza de las diferencias, como ya nos indicaba Fraser, para no caer en la mera celebración ingenua de la pluralidad sin dar cuenta de los conflictos.

De hecho, el asunto de las diferencias reintroduce el hecho del conflicto como nudo de la política frente a la ficción liberal del consenso y de la tolerancia frente a las diferencias mientras estas se avengan a vergonzosamente retirarse al ámbito de la privacidad. El problema es ahora no sólo salvable mediante procedimientos escudados en lo «razonable», sino en una cultura política que ya ha hecho sus elecciones axiológicas y ha jerarquizado los valores de acuerdo con el statu quo. El asunto aquí es, pues, el del poder y la opresión analizados desde una óptica cultural y simbólica. Las coimplicaciones entre desigualdades y diferencias tejen un tapiz enrevesado. Aquí emerge el principal escollo: los partidarios de la medicina única de la redistribución -la izquierda clásica- vislumbran la desaparición de las diferencias culturales en cuanto cese la desigualdad socioeconómica. Tan sólo contaríamos con individuos distintos, pero no con grupos de individuos con algo en común. Los partidarios de la medicina del reconocimiento pretenden la salvaguarda y el respeto de las diferencias que quedarían más allá o más acá de la desigualdad. Las tensiones éstán servidas al atender a las mediaciones y deslizamientos entre identidades individuales y colectivas.

\section{PERCEPCIÓN SOCIAL: INDIVIDUOS Y GRUPOS}

Más allá de la acotación limitada de reconocimiento de las diferencias, se nos plantea el problema de que lo que exigimos es reconocimiento de 
las personas sin obligarlas a hacer abstracción de su identidad -en la que se contienen atribuciones diferenciadas- al presentarse como ciudadanos de pleno derecho en el ámbito político. El requisito normativo no es suficiente sino que se hace sentir la necesidad de un "correcto" trato axiológico, de no desvalorización, de aquellos y aquellas que no comulguen con el modelo patrón de ciudadano. La intelección individualista de la política hace que nos equivoquemos a menudo. A los individuos no los juzgamos por sí mismos como el mito del mérito nos ha hecho pensar sino que, muchas veces a pesar nuestro, los juzgamos por sus filiaciones y pertenencias. No vemos, no percibimos ciudadanos en abstracto, sino que vemos mujeres y hombres, negros y blancos, nativos y extranjeros. Poner sobre el tapete los modos en los que se concreta nuestra percepción social de los otros y otras es el primer paso para que el reconocimiento de las personas opere efectivamente tal como debiera. Más que hacer abstracción de las diferencias habrá que colocarlas en su justo lugar tras deliberar sobre sus «efectos» sociales concretos.

De tratar con las escurridizas mediaciones entre los grupos y los individuos no nos salva nadie porque el argumento decisivo es que los vectores de opresión social, que son absolutamente pertinentes para una teoría de la justicia, pasan por su dirigirse a grupos determinados en función de algunas de sus características: sexo, raza, cultura, orientación sexual, etc. El asunto candente hoy de la ley de extanjería pasa por la percepción social del que procede de otro país, tiene otra cultura, una pigmentación de la piel tirando a oscura y, además, es pobre como candidato no suficientemente cualificado para ingresar en la ciudadanía. Este no es otro que el lado oscuro de los derechos humanos que valen para los ciudadanos, ahora comunitarios, y no para los meros seres humanos desprovistos de papeles y nacionalidad que llegan a las costas de nuestro país. Hannah Arendt ya lo señalaba al contemplar la emergencia de uno de los fenómenos claves para entender el siglo que ha acabado: los refugiados. La falta de patria conducía y aún conduce a la denegación de humanidad que no tiene otra traducción posible que la de ciudadanía. Aquí bregamos con los límites hacia fuera de las bondades o maldades de la comunidad ciudadana $x$.

El reconocimiento - de las personas y de sus diferencias- no puede ser una política entre otras políticas. Es, sin más, la condición de posibilidad de toda política que se autoconciba como radicalmente democrática y mínimamente realista, esto es, que no pretenda negar «el antagonismo, la violencia, el poder y la represión» que siguen anidando entre nosotros por decirlo con Chantal Mouffe. El ámbito de la política se sustenta en exclusiones no de individuos, sino de grupos. Los grupos 
sufren la opresión -desigualdad y/o humillación- en las carnes de sus individuos, pero estos individuos son marginados y excluidos en relación a la percepción social de su pertenencia al grupo (deseada o no). Tratar de grupos, más allá de la ontología individualista liberal, es ahora básico si queremos detectar los fenómenos de opresión que no sólo se manifiestan en un plano socio-económico sino también cultural, en el más amplio sentido del término que es relativo a las interracciones de la vida cotidiana. La utopía consensual liberal ha tenido como efecto el oscurecer la naturaleza conflictiva y desgarrada del tejido social y las diferencias de instalación en ese tejido. Si Hegel ponía contra a la pared al delincuente que traicionaba la comunidad, Marx percibió la hipocresía de la comunidad, desgarrada por la explotación y la miseria de unos frente a otros, y, sin embargo, destinada a la reconciliación en lo universal.

Los debates sobre las políticas del reconocimiento nos llevan a repensar los modos actuales de la opresión social. Aunque muchos de los viejos análisis hayan quedado inservibles ante los nuevos fenómenos, la entraña moral de analizar y denunciar los vectores de desigualdad no ha caducado.

A modo de conclusión sólo quiero aludir a alternativas políticas que, a mi parecer, merecen atención crítica por ponerse al servicio de la salvaguarda de la condición del reconocimiento como fundante del ingreso en el ámbito político. En primer lugar, frente a la invisibilidad o infrarrepresentación de determinados grupos la llamada «política de la presencia» que, por ejemplo, justifica demandas como la de la democracia paritaria, parece inexcusable. Si no se «está», dificilmente se pueden corregir los maltratos normativos y axiológicos a los que antes me refería. En segundo lugar, debemos dar más juego a las «contra-esferas públicas» que desafíen la cultura política establecida al tiempo que ofrecen alternativas a las definiciones vigentes. En tercer lugar, y, en relación con lo anterior, debe darse cauce libre a políticas de neutralización de los estereotipos negativos sobre diversos grupos estigmatizados. Por último, debemos profundizar en propuestas pragmáticas reales en las que se atienda a la corrección de las desigualdades de poder que impregnan el diálogo supuestamente imparcial y racional para así poder neutralizarlas de manera efectiva. Sólo así daremos a todos, en el marco de una democracia comunicativa, la posibilidad de ser vistos y oídos en un espacio público que es «espacio de aparición» tal como consignaba Arendt. No debemos olvidar que la propia voz se articula colectivamente, en el debate público, por eso los grupos oprimidos deben generarla hacia dentro, entre los insiders, para, después, abrirse a la posibilidad 
crítica y de redefinición de las mismas identidades a través del concurso de otras perspectivas: las de los outsiders. El reconocimiento de la titularidad política de los otros, de los diferentes, se convierte así en aspecto fundante de la política y no en una política más.

\section{REFERENCIAS BIBLIOGRÁFICAS}

FRASER, N. 1997: Justice Interruptus. London: Routledge.

HONNETH, A. 1996: La lucha por el reconocimiento. Barcelona: Gedisa.

HONNETH, A. 1998: «Reconocimiento y obligaciones morales». Revista Internacional de Filosofía Política, 8, pp. 5-17.

MARGALIT, A. 1997: La sociedad decente. Barcelona: Paidós.

TAYLOR, C. 1994: «The Politics of Recognition», en A. Gutman (ed.), Multiculturalism. Princeton: Princeton University Press.

YOUNG, I. M. 1990: Justice and the Politics of Difference. Princeton: Princeton University Press.

YOUNG, I. 1997: Intersecting Voices. Princeton: Princeton University Press. 